\title{
INITIAL EVALUATION OF A PULSED WHITE SPECTRUM NEUTRON GENERATOR FOR EXPLOSIVE DETECTION
}

\author{
Michael J. King, Gill T. Miller, Jani Reijonen, Qing Ji, Nord Andresen, Frederic Gicquel, Taneli \\ Kalvas, Ka-Ngo Leung and Joe Kwan
}

\begin{abstract}
Successful explosive material detection in luggage and similar sized containers is a critical issue in securing the safety of all airline passengers. Tensor Technology Inc. has recently developed a methodology that will detect explosive compounds with pulsed fast neutron transmission spectroscopy. In this scheme, tritium beams will be used to generate neutrons with a broad energy spectrum as governed by the $T(t, 2 n) 4 \mathrm{He}$ fission reaction that produces $0-9 \mathrm{MeV}$ neutrons. Lawrence Berkeley National Laboratory, in collaboration with Tensor Technology Inc., has designed and fabricated a pulsed whitespectrum neutron source for this application. The specifications of the neutron source are demanding and stringent due to the requirements of high yield and fast pulsing neutron emission, and sealed tube, tritium operation. In a unique co-axial geometry, the ion source uses ten parallel $r f$ induction antennas to externally couple power into a toroidal discharge chamber. There are 20 ion beam extraction slits and 3 concentric electrode rings to shape and accelerate the ion beam into a titanium cone target. Fast neutron pulses are created by using a set of parallel-plate deflectors switching between \pm 750 volts and deflecting the ion beams across a narrow slit. The generator is expected to achieve 5 ns neutron pulses at tritium ion beam energies between 80 - 120 $\mathrm{kV}$. First experiments demonstrated ion source operation and successful beam pulsing.
\end{abstract}

Index Terms-Explosive Detection, Neutron sources, PFNTS, RF Induction Ion Source, Tritium

\section{INTRODUCTION}

$\mathrm{F}$ or the past several years, Tensor Technology Inc. has been developing a method for the detection of explosive materials in airport luggage.[1] By interrogating an object with a pulsed, white energy spectrum neutrons and then comparing

Manuscript received June 30, 2008. This work is being supported by Tensor Technology Inc. under contract number LB05-001379 and Lawrence Berkeley National Laboritory under the U.S. Department of Energy, Contract DEAC02-05CH11231.

King J. Michael is with the Lawrence Berkeley National Laboratory, Berkeley, CA 94720 USA (510-495-2774; fax: 510-486-5105; e-mail: mjking@ lbl.gov).

Reijonen Jani, was with Lawrence Berkeley National Laboratory, Berkeley, CA 94720 USA. He is now with Schlumberger PTC, Princeton Junction, NJ 08550 USA (e-mail: JReijonen@princeton.oilfield.slb.com). Miller, Gill is with Tensor Technology Inc., Madison, AL 35758 USA the change in the neutron spectrum with and without that object, the presence of key elements can be determined. This technique involves neutron transmission spectroscopy where by the neutron attenuation can be determined as a function of energy using time of flight analysis. By breaking down the attenuation data into a set of linear equations for each relevant element and all neutron energies, the number density of each element can be found.[2]

Sources of pulsed white spectrum neutrons are typically created by accelerating deuterium ions up to $\mathrm{MeV}$ energies on a thick Be target, thus requiring a large accelerator.[3] An alternative source of a broad energy of neutrons is the tritiumtritium fusion reaction, $\mathrm{T}(\mathrm{t}, 2 \mathrm{n}) 4 \mathrm{He}$, which creates a flat neutron energy spectrum from $\sim 0.5 \mathrm{MeV}$ up to $9 \mathrm{MeV}$. Recently, the Lawrence Berkeley National Laboratory (LBNL) has developed a pulsed T-T neutron generator from its compact radio-frequency (rf) induction ion source and fast ion beam pulsing technologies. The evaluation of the neutron generator has been separated into two measurement phases: characterization of beam current and detection of neutron pulses. Described here is the first phase effort to generate and confirm the fast current beam pulse on target.

\section{NEUTRON SOURCE REQUIREMENTS}

A unique neutron generator is required for the successful application of pulsed fast neutron transmission spectroscopy (PFNTS) to the screening of luggage in airports. The neutron generator needs to produce a short neutron pulse and emit a high-yield broad energy spectrum of neutrons from a point source. In order to facilitate the integration of the explosives detection system into existing airport facilities, the generator and the radiation shielding also needs to be compact to minimize space requirements and foot-print.

Since time-of-flight measurement is used to determine the neutron energy after transmission through the object, a short neutron pulse width is required to provide the needed energy resolution. For the Tensor algorithm, $\sim 50 \mathrm{keV}$ energy resolution is necessary to distinguish the carbon attenuation resonances that occur between $1-3 \mathrm{MeV}$.[4] A 5 ns neutron pulse width and a $6 \mathrm{~m}$ source-to-detector distance generates an acceptable $\sim \pm 2 \%$ energy spread for a $3 \mathrm{MeV}$ transmission 
neutron.

It is also important that the neutrons be emitted from a small "point"-source, for good spatial resolution. The virtual "point" neutron source is created by using a cone shaped target that is $2 \mathrm{~cm}$ long and $5 \mathrm{~mm}$ in diameter and placing the object on axis of the target. In this way, a virtual beam spot size of $\sim 5 \mathrm{~mm}$ can be achieved with high enough surface area for adequate target cooling.

To accommodate the high throughput of luggage at airports, it is important for the scanning of luggage to be done quickly and efficiently. A high-yield neutron source $\sim 10^{10} \mathrm{n} / \mathrm{s}$ can fully scan an LD3 container filled with electronics in $\sim 20$ seconds.[4] Finally, the essential requirement is the generation of a broad neutron energy spectrum to take advantage of the energy dependent differences in attenuation for different elements.

The requirements for the neutron generator are listed in the table below.

Table 1. Generator Requirements

\begin{tabular}{|c|c|}
\hline Neutron Yield (peak) & $>10^{10}(\mathrm{n} / \mathrm{sec})$ \\
\hline Neutron Pulse Width & $<5(\mathrm{~ns})$ \\
\hline Target Diameter & $5(\mathrm{~mm})$ \\
\hline Generator Footprint & $20^{\prime \prime} \times 37 '$ \\
\hline
\end{tabular}

\section{NEUTRON GENERATOR OPERATION}

In a neutron generator, an ion beam is extracted from an ion source and accelerated onto a neutron production target. The neutron generator design described here incorporates a coaxial geometry and sealed tube construction for radioactive tritium gas operation. The ions are accelerated from a 13.56 $\mathrm{MHz}$ rf-driven external-antenna induction ion source towards the target. A water-cooled external antenna with actively water-cooled RF-window design was chosen in order to minimize the risk of cooling water mixing with tritium gas.

In a sealed generator, the gas pressure is the same throughout the vacuum envelope so the ion source must operate at the low pressure required for operating the high voltage sections without HV breakdowns. This was achieved with a set of planar, spiral antennas that allowed operation at $<$ 10 mTorr of pressure (see section V.).

The titanium target is beam-loaded. Titanium is a well established target material that forms a tridite wtih a $\mathrm{Ti}$ : T ratio of up to $1: 2$.[5] The expected neutron yield for a conservative $1: 1$ ratio at a beam energy of $120 \mathrm{kV}$ is $8 * 10^{11} \mathrm{n} / \mathrm{C}$.

\section{BEAM TRANSPORT}

The electrode system consists of three sections. The first section extracts the ions from the source, accelerates them to $30 \mathrm{keV}$, and focuses the beam for the collimator slit. The middle section includes the parallel plates and the grounded collimator. The parallel plates are designed to switch between $\pm 750 \mathrm{~V}$ for a total potential difference of $1500 \mathrm{~V}$. In the third section, the target and shroud are located in the center of the generator at a potential of $-80 \mathrm{kV}$ for the main acceleration resulting in a total beam energy of $110 \mathrm{keV}$. The reason for switching the beam at low energy is twofold: one, a lower sweeping voltage can be used to deflect the beam and second, while the beam is intercepted by the collimator the low energy minimizes the neutron generation during off-pulse. A CAD drawing of an exploded view of the electrodes is shown in Fig. 1.

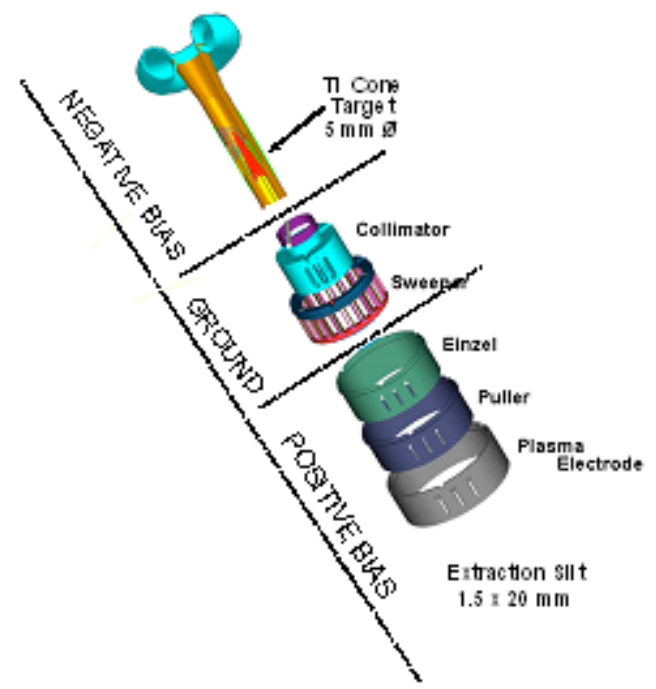

Fig. 1. Exploded CAD drawing of concentric electrodes, parallel plate sweeper and target separated and categorized by voltage potential.

The beam is extracted at the plasma electrode from 20 slits, which are $1.5 \mathrm{~mm}$ by $20 \mathrm{~mm}$. Once the ion beam leaves the plasma electrode, the puller and einzel electrodes shape and focus the beam. As the beam reaches the first grounded electrode, it is swept across a slit in the collimator by a pair of electrostatic dipole plates. The length of the beam pulse created after the collimator depends on the rise-time of the voltage sweep at the parallel plates and the width of the beam. $[6,7]$

The fast high voltage switching is controlled by a 3 ns risetime driver designed at the Lawrence Berkeley National Laboratory.[8] The rise time, which is limited by the capacitance of the parallel plates, was measured to be $12 \mathrm{~ns}$. This is expected to produce a 4 ns neutron pulse [8], which is below the specification of $5 \mathrm{~ns}$. Finally, the beam passes through the secondary electron suppression shroud that is biased below the target potential by the RC-constant associated with the shroud/target capacitance and the resistivity of the low conductance cooling water.

\section{ION SOURCE}

For a mechanically robust ion source construction, an innovative circular sapphire RF-window design, without the use of magnetic Kovar, was developed.[8] These copper framed sapphire windows were manufactured by Thermo Fusion. A 2.5 turn loosely wound planar spiral antenna was placed on each window. The antennas were made out of $1 / 8$ " diameter water-cooled copper tubing and had an inductance of approximately $0.15 \mathrm{uH}$. In order to confirm the rf coupling, beam current and ion species measurements were done for an 
ion source with one window and one antenna. The results are shown in Fig. 2 and 3.

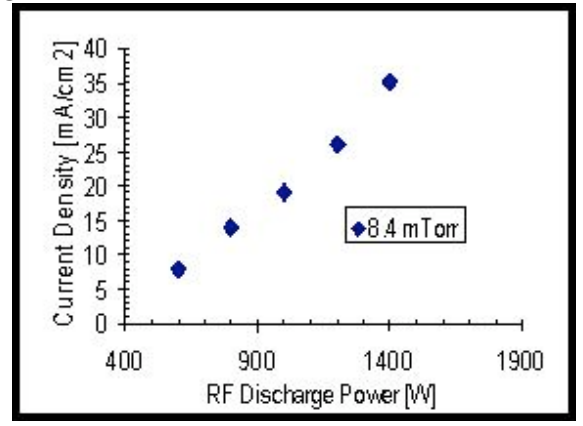

Fig. 2. Current density measurement as a function of rf power.

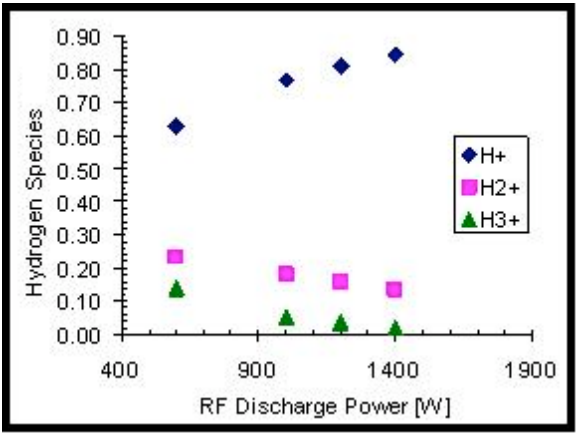

Fig. 3. Hydrogen species measurement as a function of $\mathrm{rf}$ power. The $\mathrm{H}+$ species increases as a function of rf power.

At $1 \mathrm{~kW}$ of rf power, the measured current density was 18 $\mathrm{mA} / \mathrm{cm}^{2}$ and the $\mathrm{H}+$ species fraction was around $80 \%$. The operational gas pressure of $8.4 \mathrm{mTorr}$ is expected to be sufficiently low to avoid high voltage breakdowns in the accelerating section.

Before the design was finalized, a prototype ion source was fabricated. Ten quartz windows placed in a straight line for easier configuration of the rf antennas is shown in Fig 4.

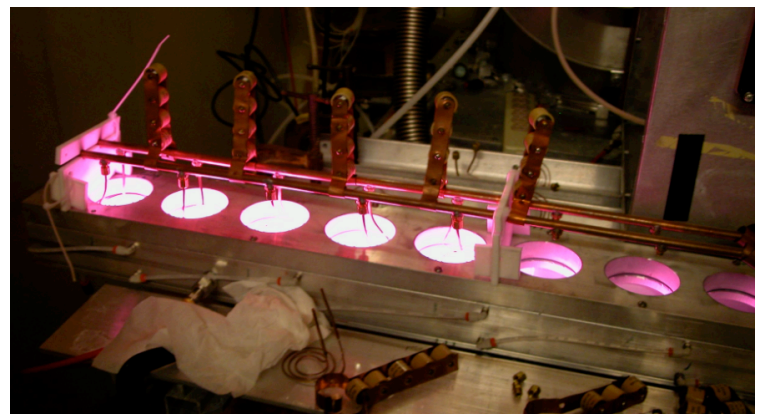

Fig. 4. Prototype 10 window ion source fabricated for ignition uniformity testing. 5 windows are equipped with $\mathrm{rf}$ antennas and uniformly lit. Capacitors are placed in parallel to the antennas.

In the initial testing of the straight ion source prototype with multiple rf induction antennas connected, the discharge luminosity of the plasma varied from antenna to antenna. Even though the plasma had already transitioned from a capacitive to an inductive discharge (from a dim to brighter plasma), variation in brightness was still noticeable. Non-uniformity in luminosity corresponds to differences in plasma density between the windows. Because the ion beam focusing is similar for each beamlet, the varying plasma density could easily lead to incorrect focusing and ultimately arcing and breakdown issues at the accelerator structure.

It was discovered that by placing a set of $\sim 300 \mathrm{pF}$ capacitors in parallel with an antenna that had weakly lit plasma, the plasma in that window would become brighter. By maneuvering the capacitors along the main antenna bus a uniform plasma could be created among all windows. With 5 $\mathrm{kW}$ of $\mathrm{rf}$ power, and a maximum of 5 antennas in parallel we were able to achieve uniform plasma brightness. Fig. 5 shows the circuit diagram that was analyzed with RFSIM99.[9] The circuit was matched to the $50 \mathrm{ohm}$ impedance of the $\mathrm{rf}$ generator, with an estimated plasma impedance of $3 \mathrm{ohms}$.

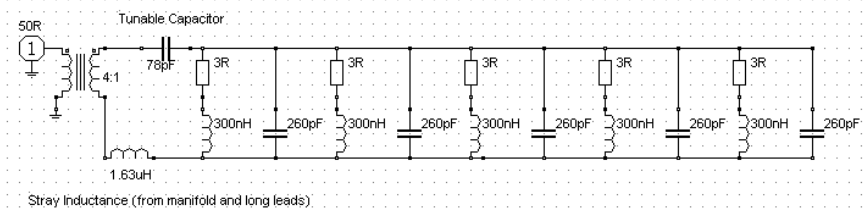

Fig. 5 Schematic of the antenna and capacitor in parallel configuration.

Thus by splitting the ten windows into two rf circuits of 5 parallel rf antenna, ten uniformly lit plasmas were obtained. On average, each plasma absorbs $1 \mathrm{~kW}$ of $\mathrm{rf}$ power. In the final design, shown in Fig 6, the circular ion source is uniformly ignited and a close-up view of an antenna and sapphire window is shown.

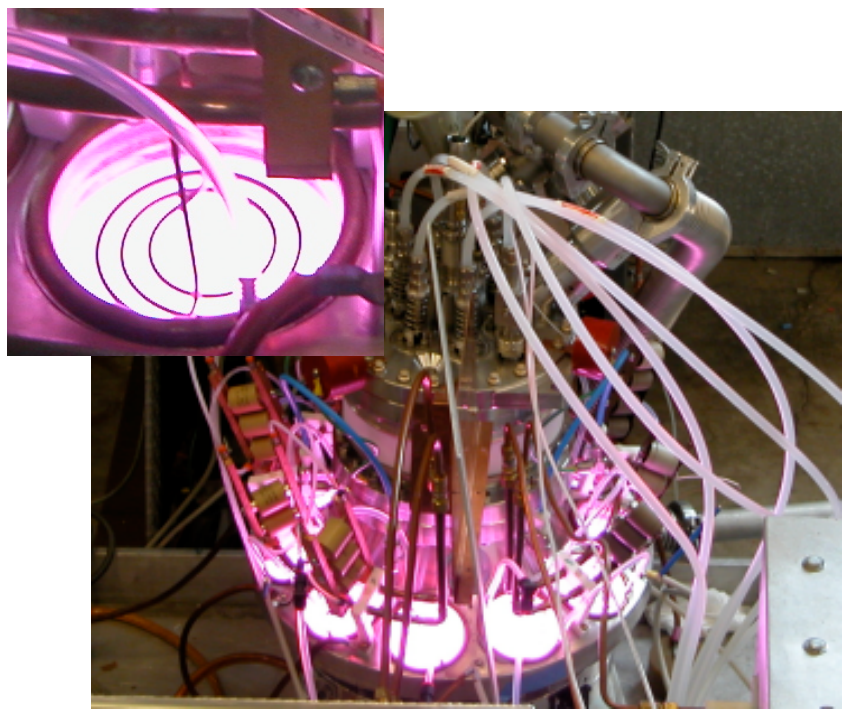

Fig. 6. Enlarged view of one antenna and its sapphire ion source window. Below is the actual neutron generator being tested with all ten windows lit.

The plasma ignites at 80 mTorr and $2000 \mathrm{~W}$ of continuous rf power. Stable operation of the source is achieved down to 8 mTorr of gas pressure.

\section{CURRENT MEASUREMENTS}

Fast current waveforms with nanosecond rise times are difficult to measure accurately with the use of a shunt resistor or Rogowski transducer coil due to the presence of RF noise. In order to alleviate the complexity of measuring a fast current 
pulse on target during optimization of electrode voltages, the sweeping rise time was slowed considerably by replacing the switch with a simple $60 \mathrm{~Hz}$ variable transformer. The $60 \mathrm{~Hz}$ output was placed in series with a high voltage transformer, producing a 760 volt peak bias with an $8 \mathrm{~ms}$ rise time. With a longer sweeping rise time, the current pulse shape could be easily observed on the oscilloscope. In Fig. 7, the oscilloscope trace shows three current pulses corresponding to three plasma electrode biases and the $60 \mathrm{~Hz}$ sweeping voltage. The data was taken with the ion source running with $2000 \mathrm{~W}$ of RF power and at half capacity with only 5 out of the 10 windows being used for plasma generation.

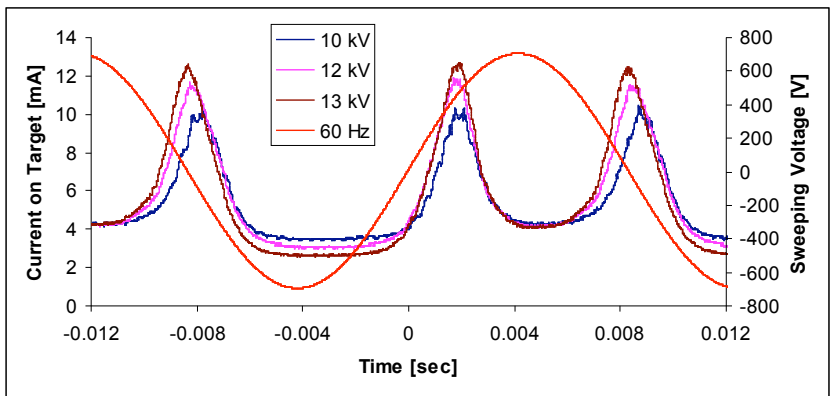

Fig. 7. Current on target and $60 \mathrm{~Hz}$ sweeping voltage as a function of time.

The measurements demonstrated that the beam behaved properly. The electrodes accelerated the beam without any breakdowns and the parallel plate voltage sweeping created a current pulse on a grounded target. A $12 \mathrm{~mA}$ current pulse is observed, with a $13 \mathrm{kV}$ plasma electrode (extraction) voltage, as the sweeping bias moves through zero potential. With the 8 ms rise time created by the $60 \mathrm{~Hz}$ sweep, a $3 \mathrm{~ms}$ current pulse width was recorded on target. The beam width is larger than 2 $\mathrm{mm}$ (the slit width), because the pulse shape does not have a flat top representing constant current through the slit. Previous simulations showed a $2 \mathrm{~mm}$ beam width was achievable at the collimator with $30 \mathrm{kV}$ of extraction voltage and a well matched plasma density.[7]

Previous fast pulse measurements showed a potential problem with switched voltage ringing.[8] This causes the beam to oscillate and produce a distorted current pulse structure. The ringing was eliminated by adding $50 \mathrm{ohm}$ resistors in series with the parallel plates.

The two scope traces shown in Fig. 8 (top) show a 12 ns rise time that generates a 4 ns pulse width on target. After elimination of the ringing with added resistors, the rise time slowed to $40 \mathrm{~ns}$ allowing for a $12 \mathrm{~ns}$ current pulse width. To be noted, the pulse width can be further shortened by increasing the sweeping voltage while keeping the rise time the same.
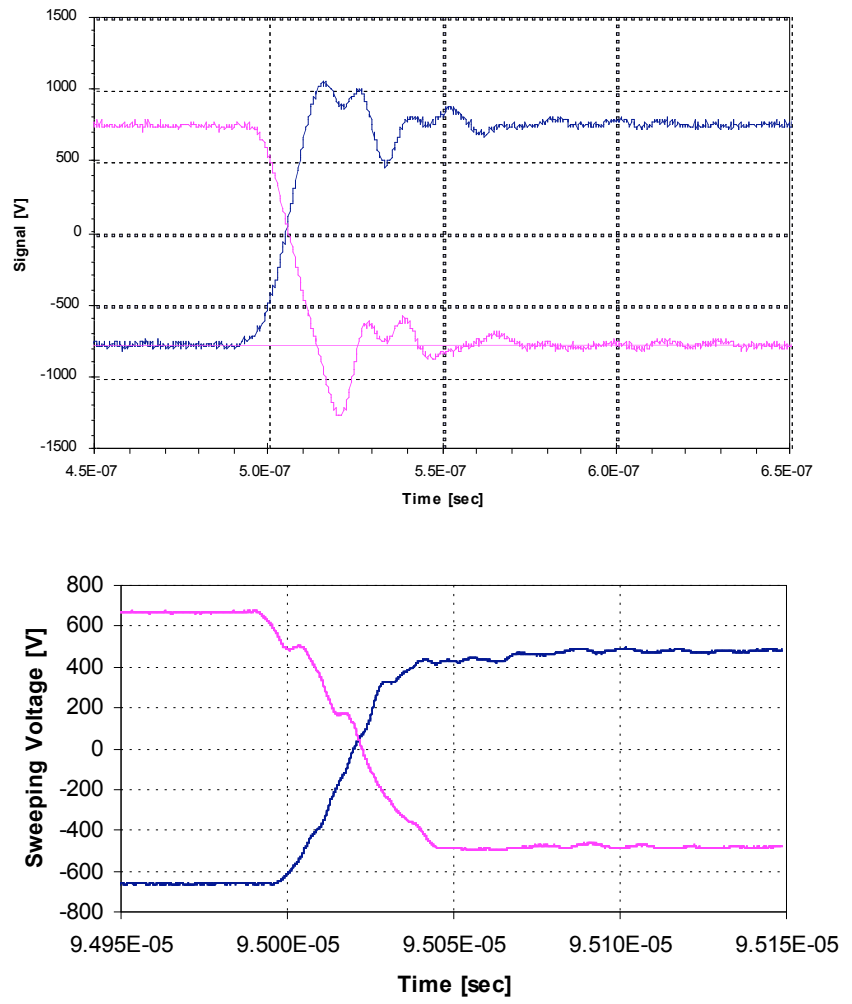

Fig. 8. A comparison of rise times and pulse widths where the top figure shows a faster rise time but large high voltage ringing. The bottom figure shows the absence of ringing although the rise time has increased. Both positive and negative rise times are shown.

Even at the slower rise time of $40 \mathrm{~ns}$, the current pulse on target was still too difficult to observe on the scope because of rf noise and capacitively coupled ringing on the target cable. In order to measure the current pulse on target, the rise time was further slowed to 200 ns, shown in Fig. 9, by placing capacitors between the switching electrodes and ground.

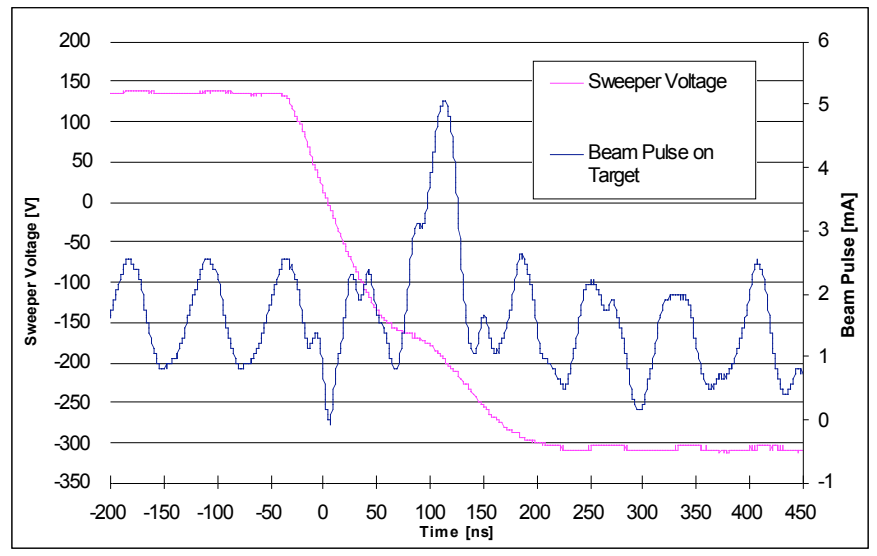

Fig. 9. Sweeper voltage and current pulse on target as a function of time. A $200 \mathrm{~ns}$ rise time generates a $70 \mathrm{~ns}$ current pulse width on target.

\section{CONCLUSION}

The initial testing of the pulsed white spectrum neutron generator has been completed. The ten window ion source has achieved uniform discharge luminosity by the placement of 
capacitors in parallel with the induction antenna loops. We have successfully recorded current pulses on target. Our latest results show $36.7 \mathrm{~mA}$ of peak current on target with $3 \mathrm{~kW}$ of rf power and 5 operating windows using the $60 \mathrm{~Hz}$ sweeping. This indicates that $200 \mathrm{~mA}$ can be obtainable with $\sim 16 \mathrm{~kW}$ of rf power by linearly scaling the current and rf power.

High voltage conditioning of the accelerator structure has been completed. By setting the gas pressure at $10 \mathrm{mTorr}$ and closing the pumping valve for a "sealed" operating condition, the target high voltage held $80 \mathrm{kV}$ without sparking, while the plasma electrode withstood $30 \mathrm{kV}$. With pumping, we were able to accelerate a $20 \mathrm{~mA}$ beam on target with the plasma electrode and target at 20 and $70 \mathrm{kV}$ respectively.

The 4 ns current pulse had been purposely slowed to $70 \mathrm{~ns}$ to avoid target current measurement complications arising from rf noise and capacitively coupled ringing from the fast high voltage switching. Without the capacitors to slow the rise time of the switches the rise time is $40 \mathrm{~ns}$ without noticeable ringing. This would generate a 12 ns current pulse on target with \pm 750 deflection voltages. A faster pulse can be attained by increasing the deflection voltage on the parallel plates.

In the next testing phases it is planned to operate the generator with deuterium gas and measure the produced neutrons. These measurements will allow a direct determination of pulse structure and length without being affected by rf noise. After these tests, it is planned to UHV process the generator and bake it at $120^{\circ} \mathrm{C}$ with argon gas flow in preparation for sealed operation using tritium gas.

\section{ACKNOWLEDGMENT}

The authors would like to thank Jim Ellis, Steve Wilde, Tom McVeigh and Paul Wong, for technical support and Will Waldron and James Galvin for their expertise with current measurement and fast switching devices.

\section{REFERENCES}

[1] T. G. Miller, "Use of neutron tomography for airline security," in Substance Identification Analytics, Innsbruck, Austria, 1994, pp. 204-217.

[2] T. G. Miller and R. A. Krauss, "Substance identification using neutron transmission," in Law Enforcement Technologies: Identification Technologies and Traffic Safety, Munich, Germany, 1995, pp. 14-24.

[3] D. L. Smith and J. W. Meadows, "A facility for high intensity irradiation using thick-target sources at Argonne Fast-Neutron Generator," Argonne National Laboratory 1986.

[4] J. Ellis, "Personal Communication," 2008.

[5] J. Csikai, "Handbook of fast neutron generators," vol. I, 1987.

[6] S. K. Hahto, S. T. Hahto, K. N. Leung, J. Reijonen, T. G. Miller, and P. K. Van Staagen, "Fast ion beam chopping system for neutron generators," Review of Scientific Instruments, vol. 76, pp. -, Feb 2005.

[7] T. Kalvas, S. K. Hahto, F. Gicquel, M. King, J. H. Vainionpaa, J. Reijonen, et al., "Fast slit-beam extraction and chopping for neutron generator," Review of Scientific Instruments, vol. 77, pp. 03B904-3, 2006.

[8] J. Reijonen, N. Andresen, F. Gicquel, R. Gough, M. King, T. Kalvas, et al., "Development of advanced neutron/gamma generators for imaging and active interrogation applications," in Optics and Photonics in Global Homeland Security III, Orlando, FL, USA, 2007, pp. 65401P-12.

[9] S. Hyde, "RFSIM99," 1.05 ed: HyDesign Ltd, 1999, p. Circuit Analysis. 